\title{
Comparison of numerical simulations and observations of helioseismic MHD waves in sunspots
}

\author{
K. V. Parchevsky ${ }^{1}$, J. Zhao ${ }^{1}$, A. G. Kosovichev ${ }^{1}$ and M. Rempel ${ }^{2}$ \\ ${ }^{1}$ Hansen Experimental Physics Laboratory, Stanford University, \\ Stanford, CA 94305, USA \\ email: kparchevsky@solar.stanford.edu \\ ${ }^{2} \mathrm{HAO} / \mathrm{NCAR}$, Boulder, CO 80307, USA \\ email: rempel@ucar.edu
}

\begin{abstract}
Numerical 3D simulations of MHD waves in magnetized regions with background flows are very important for the understanding of propagation and transformation of waves in sunspots. Such simulations provide artificial data for testing and calibration of helioseismic techniques used for analysis of data from space missions SOHO/MDI, SDO/HMI, and HINODE. We compare with helioseismic observations results of numerical simulations of MHD waves in different models of sunspots. The simulations of waves excited by a localized source provide a detailed picture of the interaction of the MHD waves with the magnetic field and background flows (deformation of the waveform, wave transformation, amplitude variations and anisotropy). The observed cross-covariance function represents an effective Green's function of helioseismic waves. As an initial step, we compare it with simulations of waves generated by a localized source. More thorough analysis implies using multiple sources and comparison of the observed and simulated cross-covariance functions. We plan to do such calculations in the nearest future. Both, the simulations and observations show that the wavefront inside the sunspot travels ahead of a reference "quiet Sun" wavefront, when the wave enters the sunspot. However, when the wave passes the sunspot, the time lag between the wavefronts becomes unnoticeable.
\end{abstract}

Keywords. Helioseismology, MHD, sunspots

\section{Introduction}

Understanding of acoustics wave generation, propagation, and scattering in the convective zone of the Sun is one of the high-priority tasks of helioseismology. The commonly used acoustic ray theory is not applicable near the top turning point (near the photosphere) where the background model changes quickly due to large gradients of pressure and density, and wave effects must be taken into account. Different types of MHD waves (fast, slow, Alfven) and magneto-gravity wave exist in magnetized regions on the Sun. Their interference, transformation, and reflection from the top boundary creates very complicated picture. In this situation numerical simulations of MHD waves in sunspots are important for understanding the behavior of the acoustic waves in the complicated solar environment. They also provide artificial data for testing and calibrating the helioseismic measurement and inversion techniques used for processing data from space missions SOHO, Hinode and SDO.

\section{MHD wave modeling}

Propagation of waves inside the Sun, in the presence of magnetic fields and velocities in the background state, is described by linearized MHD equations (see Parchevsky \& 

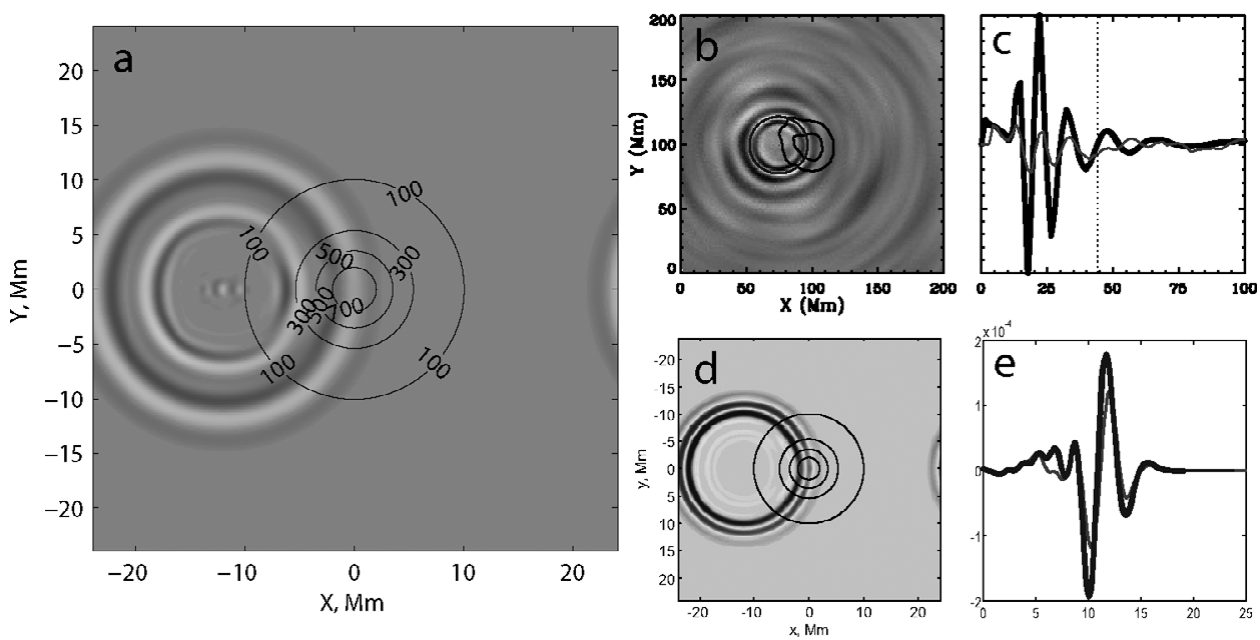

Figure 1. Snapshot of the $z$-component of the velocity at the level of the photosphere (panel a). Cross-correlation obtained from SOHO/MDI observations by J. Zhao for active region AR9787 (panel b) and a snapshot of the $p$-mode front (panel d) from simulations in the magnetostatic sunspot model. The light and heavy curves (panels $\mathrm{c}$ and e) represent cuts through the sunspot and the quiet Sun region respectively.

Kosovichev (2009) for details):

$$
\begin{aligned}
\frac{\partial \rho}{\partial t}= & -\nabla \cdot \boldsymbol{m}^{\prime}-\nabla \cdot\left(\rho^{\prime} \boldsymbol{V}_{0}\right) \\
\frac{\partial \boldsymbol{m}^{\prime}}{\partial t}= & -\nabla p^{\prime}-\left(\boldsymbol{m}^{\prime} \cdot \nabla\right) \boldsymbol{V}_{0}-\left(\boldsymbol{V}_{0} \cdot \nabla\right) \boldsymbol{m}^{\prime}-\boldsymbol{m}^{\prime} \nabla \cdot \boldsymbol{V}_{0}-\rho^{\prime}\left(\boldsymbol{V}_{0} \cdot \nabla\right) \boldsymbol{V}_{0} \\
& -\frac{1}{4 \pi} \nabla\left(\boldsymbol{B}_{0} \cdot \boldsymbol{B}^{\prime}\right)+\frac{1}{4 \pi}\left[\left(\boldsymbol{B}_{0} \cdot \nabla\right) \boldsymbol{B}^{\prime}+\left(\boldsymbol{B}^{\prime} \cdot \nabla\right) \boldsymbol{B}_{0}\right]+\rho^{\prime} \boldsymbol{g}_{0}+\boldsymbol{S}(\boldsymbol{r}, t) \\
\frac{\partial \boldsymbol{B}^{\prime}}{\partial t}= & \nabla \times\left(\frac{\boldsymbol{m}^{\prime}}{\rho_{0}} \times \boldsymbol{B}_{0}\right)+\nabla \times\left(\boldsymbol{V}_{0} \times \boldsymbol{B}^{\prime}\right) \\
\frac{\partial p^{\prime}}{\partial t}= & c_{s}^{2} \frac{\partial \rho}{\partial t}-c_{s}^{2} \boldsymbol{m}^{\prime} \cdot\left(\frac{\nabla p_{0}}{\Gamma_{1} p_{0}}-\frac{\nabla \rho_{0}}{\rho_{0}}\right)-c_{s}^{2} \rho_{0}\left(\frac{p^{\prime}}{p_{0}}-\frac{\rho^{\prime}}{\rho_{0}}\right) \nabla \cdot \boldsymbol{V}_{0} \\
& -\boldsymbol{V}_{0} \cdot \nabla p^{\prime}+c_{s}^{2} \boldsymbol{V}_{0} \cdot \nabla \rho^{\prime}
\end{aligned}
$$

where $\rho^{\prime}, p^{\prime}, \boldsymbol{m}^{\prime}=\rho_{0} \boldsymbol{v}^{\prime}$, and $\boldsymbol{B}^{\prime}$ are the perturbations of the density, pressure, momentum, and magnetic field respectively. Quantities $p_{0}, \rho_{0}, c_{s}, \boldsymbol{g}_{0}, \boldsymbol{V}_{0}$, and $\boldsymbol{B}_{0}$ are the background pressure, density, sound speed, gravitational acceleration, flow velocity, and the magnetic field respectively. The source term $\boldsymbol{S}=\left(0,0, S_{z}(\boldsymbol{r}, t)\right)$ represents the acoustic source, localized in space and explicitly depending on time as Rickers wavelet. This source model provides the wave spectrum, which closely resembles the solar spectrum (see details in Parchevsky \& Kosovichev (2007)).

A semi-discrete finite-difference numerical scheme is used. It easily permits to combine different versions of spatial discretization and time advancing schemes. A dispersionrelation-preserving scheme developed by Tam \& Webb (1993) is used for the spatial discretization, and a strong stability preserving Runge-Kutta scheme is used for time advancing.

The waves with frequencies lower than the acoustic cut-off frequency are reflected below the photosphere. The waves with higher frequencies pass through and penetrate 

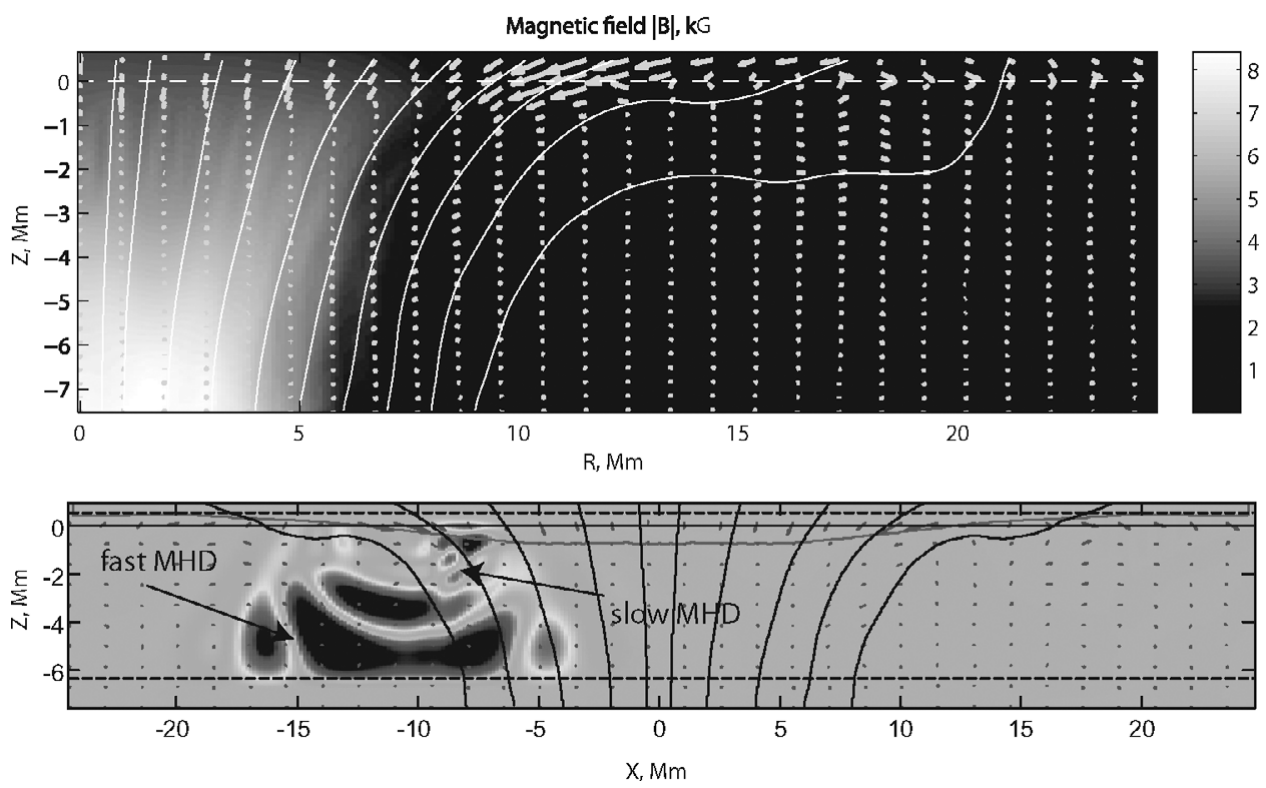

Figure 2. Magnetic field strength of the axially symmetric (angular averaged) realistic model of the sunspot with the background flows (top) obtained from numerical non-linear MHD simulations (M. Rempel). The white curves show the magnetic field lines, arrows represent the background flows. The snapshot of the model is used as the background state for the wave simulations. The bottom panel shows perturbations of the vertical momentum of the wave generated by a single source. A mixture of slow MHD and Alfven waves at the source location is observed. The rigth part of the wave front is skewed by the background flows. This skewness is not observed in simulations with magnetostatic models.

into the chromosphere. To simulate such frequency-dependent reflecting property we put the Perfectly Matched Layer (PML) at $500 \mathrm{~km}$ above the photosphere. On the Sun all waves which reach this layer propagate further to chromosphere. In simulations they will be absorbed by PML and do not pollute the computational domain. To prevent spurious reflection from the bottom of the domain we put the PML at the bottom boundary. The lateral boundary conditions are chosen to be periodic, which mimics incoming waves.

\section{Results and discussion}

Observational cross-correlation function. In this section we compare the behavior of the observational cross-correlation function inside a sunspot observed by SOHO/MDI and simulations of the wave front from a single source inside the magnetostatic sunspot model (Parchevsky et al. (2010)). The backgrouns model was provided to us by Khomenko \& Collados (2008). For both simulations and observations the $f$-mode signal has been filtered out. Qualitatively, the top and bottom panels show similar behavior. In both figures, the waves propagating through the sunspot have smaller amplitude than the waves propagating in the quiet regions. Both, simulations and observations, show that the wave front inside the sunspot travels ahead of the "quiet" wave front when the wave enters the sunspot. Both, simulations and observations, show that when the wave passes the sunspot, the time lag between wave fronts becomes unnoticeable.

Realistic sunspot model with the background flows In Figure 2 we present snapshot of the vertical momentum of MHD waves (bottom panel) in a realistic sunspot model with background flows (top panel). The background model was provided us by Rempel et al. 
(2009). In deep layers the right part of the wave front is noticeably skewed due to the background flows (the wave front in the same region of the magnetostatic model is almost symmetric). It seems that in deep layers the shape of the wave front of the fast MHD wave is affected stronger by flows than by magnetic fields.

\section{Conclusion}

Comparison of simulations for magnetostatic models of sunspots with observations shows that the observational wave forms cross-correlation functions show similarities to the behavior of the numerically simulated wave front of $p$-modes: (i) the amplitude is reduced inside the sunspot; (ii) when the wave enters the sunspot, the wave front inside the sunspot travels ahead of the wave front in the quiet region. We developed a $3 \mathrm{D}$ code for numerical simulation of MHD waves in models of sunspots in presence of the background flows. In addition to the primary fast MHD wave, used for helioseismic studies, simulations show mixture of slow MHD and Alfven waves excited by the source, and traveling along field lines into the interior (as in the magnetostatic model). The simulations reveal also a strong distortion of the wave front due to the background velocities (not present in the magnetostatic models). This code is used for simulations of stochastic oscillations excited by multiple random sources for testing local helioseismology and calibration of helioseismic inferences of the subsurface structure of sunspots.

\section{Acknowledgements}

We are grateful to E. Khomenko and M. Collados for providing us magnetostatic models of sunspots. This research was supported by NASA LWS grant NNG05GM85G to Stanford University.

\section{References}

Khomenko, E. \& Collados, M. 2008, Astrophys. J., 689, 1379

Low, B. C. 1975, Astrophys. J., 197, 251

Parchevsky, K. V. \& Kosovichev, A. G. 2007, Astrophys. J., 666, 547

Parchevsky, K. V. \& Kosovichev, A. G. 2009, Astrophys. J., 694, 573

Parchevsky, K. V., Kosovichev, A. G., Khomenko, E., Olshevsky, V., \& Collados, M. 2010, arXiv:1002.111\%v1

Pizzo, V. J. 1986, Astrophys. J., 302, 785

Rempel, M., Schüssler, M., Cameron, R. H., \& Knölker, M. 2009, Science, 325, 171

Tam, C. \& Webb, J. 1993, J. Comput. Phys. 107, 262 